\title{
IDIOPATHIC GRANULOMATOUS MASTITIS IN A PREGNANT WOMAN
}

Israel Moreira Ramos de Souza', Francisca Indira Beltrão Colaço Costa da Matta², José Ismair de Oliveira dos Santos ${ }^{1}$, Thayrone de Miranda Barreto ${ }^{1}$

${ }^{1}$ Universidade Estadual de Ciências da Saúde de Alagoas - Maceió (AL), Brazil.

${ }^{2}$ Hospital Memorial Arthur Ramos - Maceió (AL), Brazil.

Introduction: Idiopathic granulomatous mastitis (IGM) is a rare inflammatory breast disease of unknown etiology. It was described in 1972 by Kessler and Wolloch, who reported cases of granulomatous lobulitis with no established relationship with trauma, foreign body, or specific infection. This study aimed at reporting a case of IGM in a pregnant patient. Clinical case: A 36-year-old pregnant woman, with a gestational age of 27 weeks, was admitted with a complaint of right breast pain for 7 days. She denied comorbidities. Clinical examination showed a dense area in the junction of the right inner quadrants, associated with mild hyperemia without signs of fluctuation. Lymphadenopathy was not identified. Ultrasound revealed a non-homogeneous fluid of 4.9x1.7x5.2 cm in the right upper inner quadrant, with a consequent surgical approach and collection of fragments for analysis. Cultures with antibiogram and for fungi and the venereal disease research laboratory (VDRL) tested negative. Histopathological examination showed a chronic inflammatory process with abundant granulation tissue and isolated giant cells, suggesting granulomatous mastitis. Corticosteroid therapy was indicated after the birth, with prednisone $40 \mathrm{mg} /$ day for 3 months, with complete resolution of the symptoms. Discussion: Usually, IGM affects women aged 17 to 42 years. Recent history of pregnancy is common, with identified cases ranging from 2 months to 15 years after the last delivery, rarely occurring during gestation. It manifests as a breast mass of 0.5 to $9 \mathrm{~cm}$ in diameter, often unilateral and on the left side. Signs of inflammation and local lymphadenopathy can also be identified. Mammography is not ideal for detecting IGM, with findings of low specificity, and false negatives are frequent in dense breasts. The most frequent ultrasound findings are irregular hypoechoic mass and tubular hypoechoic lesion. IGM can be confirmed by detecting a histological pattern of chronic granulomatous lobulitis, non-caseating granuloma with infiltration of histiocytes, few polymorphonuclear cells, and multinucleated giant cells. Ruling out syphilis, fungi, and breast tuberculosis is important. The recommended treatment is excisional biopsy, preferably a wide excision. However, surgical management has a negative cosmetic impact and is associated with up to $50 \%$ of cases of persistence and recurrence of the disease, in addition to complications such as abscesses, fistulas, and ulceration. Corticosteroids are an effective option for controlling the disease, but active surveillance can also be chosen. Considering the above, we underline the importance of early diagnosis with appropriate scientific and technical foundations to provide a better prognosis for patients. 